\title{
Correcting palindromes in long reads after whole-genome amplification
}

\author{
Sven Warris ${ }^{1^{*}}$ (D), Elio Schijlen ${ }^{1}$, Henri van de Geest ${ }^{1,10}$, Rahulsimham Vegesna ${ }^{3,4,5}$, Thamara Hesselink', \\ Bas te Lintel Hekkert ${ }^{1}$, Gabino Sanchez Perez ${ }^{1,10}$, Paul Medvedev ${ }^{5,6,7,8}$, Kateryna D. Makova ${ }^{8,9}$ and Dick de Ridder ${ }^{2}$
}

\begin{abstract}
Background: Next-generation sequencing requires sufficient DNA to be available. If limited, whole-genome amplification is applied to generate additional amounts of DNA. Such amplification often results in many chimeric DNA fragments, in particular artificial palindromic sequences, which limit the usefulness of long sequencing reads.

Results: Here, we present Pacasus, a tool for correcting such errors. Two datasets show that it markedly improves read mapping and de novo assembly, yielding results similar to these that would be obtained with non-amplified DNA.

Conclusions: With Pacasus long-read technologies become available for sequencing targets with very small amounts of DNA, such as single cells or even single chromosomes.

Keywords: Whole-genome amplification, High molecular weight DNA, Long read sequencing, Palindromes, Chimeric reads, de novo assembly, Read mapping
\end{abstract}

\section{Background}

Modern sequencers require sufficient material to work with: the Illumina and Pacific Bioscience (PacBio) platforms prescribe at least three micrograms, but recommend at least five [1] micrograms. Long-read sequencing technologies such as those offered by PacBio and Oxford Nanopore Technology (ONT) additionally require high molecular weight (HMW) DNA as a starting material, i.e. material in which individual DNA stretches are long. In many biological settings, obtaining sufficient amounts of DNA of the required quality and length is problematic, such as in studies on single cells $[2,3]$ or single selected chromosomes [4]. To overcome this limitation DNA is amplified, starting from as little as picograms, in a process called whole-genome amplification (WGA) [5].

A major issue with the WGA process is that it introduces specific chimeric fragments $[6,7]$ consisting of one or more inverted repeats (Fig. 1), so-called palindromes. This effect is partially alleviated by de-branching, however, chimeric fragments still remain [8]. In Illumina paired-end (PE) sequencing these fragments are then sheared into small sub-fragments before library preparation, which

\footnotetext{
* Correspondence: sven.warris@wur.nl

${ }^{1}$ Applied Bioinformatics, Wageningen University and Research, Wageningen,

The Netherlands

Full list of author information is available at the end of the article
}

reduces the effect on subsequent analyses of the palindromic fragments as they will occur in only few reads. In other approaches to sequencing, however, the full fragments are used. For Illumina mate-pair (MP) sequencing, long palindromic fragments will result in pairs with incorrect directions and unpredictable insert sizes. As a result, short read MP libraries based on WGA are problematic for read mapping and de novo assembly.

Tools specifically aiming to detect and correct chimeric reads have been proposed (e.g. uchime [9]) and work well for paired-end and single-end short-read technologies.

For long reads however, the palindromic nature of the sequence hinders read mapping and renders de novo assembly highly problematic. Due to the high base-calling error rate of the long read technologies (11-38\%) [10, 11], finding and correcting these palindromic constructs in long reads cannot be done by exact string matching. Algorithms for improving long-read quality in general are available: Proovread [12], PBcR [13] and ECTools [14] use either Illumina HiSeq reads or assembled contigs based on HiSeq data to increase the quality of base calling. While Proovread can also detect chimeric fragments, it specifically aims at detecting PCR artifacts joining fragments originating from different regions in the genome. This is done by mapping HiSeq reads, assumed to be available, which do not have these chimeras. The location 


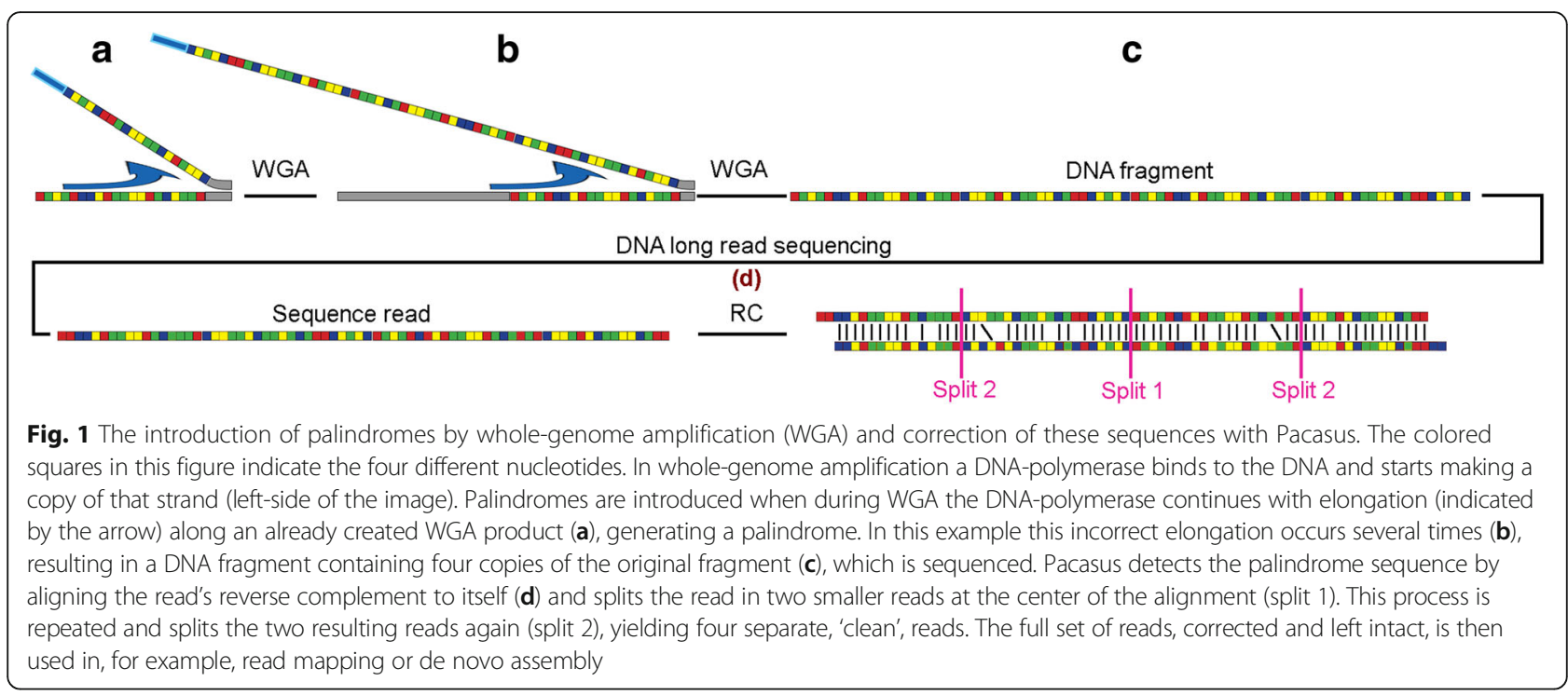

of the chimera in the long read is then detected by finding discrepancies in the short-read mappings. This approach is unfit for solving the chimeras occurring due to WGA: the HiSeq reads are based on the same fragments as the long reads and will therefore contain the same nucleotide sequence information. As a consequence of this lack of suitable methods to correct these chimeras, the use of WGA with long-read technologies was usually not advised [6], which precludes the application of long read technology to answer essential biological questions at the single-chromosome or single-cell level.

Here, we introduce a new method, Pacasus, for correcting palindromic, long, error-rich reads without the loss of nucleotide information and with only very limited impact on repeats and palindrome sequences of biological origin. The method relies on a Smith-Waterman alignment implementation called pyPaSWAS [15, 16], which supports fast processing on multicore CPUs, GPUs and Xeon Phis to detect palindromes and iteratively corrects them by splitting up reads. We demonstrate its performance on PacBio sequencing data of Arabidopsis thaliana as well as on flow-sorted gorilla $\mathrm{Y}$ chromosome data, by using the multiple displacement amplification kit REPLI-g for the amplification process.

The gorilla Y chromosome was selected because primate $\mathrm{Y}$ chromosomes are relatively short and contain many repeats, rendering them difficult to sequence and assemble. Even in one of the most complete assemblies, that of Homo sapiens, more than half of the sequence of $\mathrm{Y}$ is still unknown [17]. To obtain a higher read coverage of the gorilla Y chromosome, a recent paper [4] used flow-sorting and WGA. PacBio long reads, Illumina HiSeq PE and MP-libraries, transcriptome data and PCR sequences were used by the authors as well (Bioproject PRJNA293447). The RecoverY tool [4, 18] presented in same paper was designed to identify short reads originating from the $\mathrm{Y}$ chromosome. Based on these data, the authors created a hybrid (PacBio + HiSeq) assembly, here labeled 'GorY'. The authors also used HGAP [19] and MHAP [20] to create PacBio-only assemblies, but these resulting assemblies were of suboptimal quality. In this manuscript, we used the PacBio data after WGA generated by Tomaszkiewicz and colleagues [4] to show the benefits of correcting palindrome sequences in this data set with an improved quality of the PacBio-only de novo genome assembly.

\section{Results}

\section{Pacasus corrects many palindromic sequences found in} WGA data

To demonstrate the added value of Pacasus in the analysis of PacBio reads generated from WGA DNA samples, we applied it to several data sets of Arabidopsis thaliana and a data set of the gorilla Y chromosome [4] (Table 7). Table 1 lists the original number of reads, the number of reads that were found to have chimeras and the number of clean reads after correcting the palindromes. In the Arabidopsis samples, $40-50 \%$ of reads contained at least one palindrome, with some reads containing up to 15 (Additional file 1). This demonstrates the extent to which palindromes pose a problem in PacBio WGA data and illustrates that Pacasus effectively detects and corrects these.

Table 1 shows that Pacasus decreases the average read length of the $A$. thaliana set by about $48 \%$, i.e. preserving much of the long-range information. In the gorilla read set, $11.8 \%$ of the reads contain palindromes, less than in the A. thaliana sets. The average length of the gorilla reads before processing with Pacasus is $5468 \mathrm{~b}$, i.e. $61.2 \%$ of the average length in the total Ath-WGA data 
Table 1 Effect of correcting palindromes

\begin{tabular}{|c|c|c|c|c|c|c|}
\hline \multirow[t]{2}{*}{ Sample } & \multicolumn{2}{|l|}{ Before cleaning } & \multicolumn{2}{|c|}{ Reads with detectable palindromes } & \multicolumn{2}{|l|}{ After correcting } \\
\hline & Number of reads & Average length (b) & Number of reads & Number of reads (\%) & Number of reads & Average length (b) \\
\hline Ath-WGA1 & 462,138 & 9326 & 221,001 & 47.8 & 869,826 & 4660 \\
\hline Ath-WGA2 & 447,364 & 8544 & 195,263 & 43.6 & 769,027 & 4721 \\
\hline Ath-Ctrl & 940,162 & 5680 & 4714 & 0.5 & 938,196 & 5681 \\
\hline GorY-WGA & $3,596,236$ & 5468 & 426,188 & 11.8 & $4,546,488$ & 4234 \\
\hline
\end{tabular}

Effect of correcting palindromes on the number reads and average lengths of these reads. Note: the Ath-Ctrl shows a small increase in average read length after correction and a lower number of reads. This is because Pacasus removes very short reads from the output

set (8934b); after correcting the palindromes this is increased to $90.3 \%$ : $4234 \mathrm{~b}$ compared to $4689 \mathrm{~b}$. Pacasus finds palindromes in only in $0.5 \%$ of the reads in the non-amplified control data set, Ath-Ctrl. These reads will be a mixture of false-positives and missed/missing SMRTBell adaptors, which also cause palindromic sequences. The low number of palindromes found in the control set by Pacasus means that there is no need to perform subsequent analyses on 'Ath-Ctrl-Clean': the resulting de novo assembly for example will not be different from the one based on the original 'Ath-Ctrl' set.

The GC contents of the read sets were compared to that of the $A$. thaliana reference genome and no biases were observed for both the amplification process and the palindrome detection by Pacasus (Fig. 2).

\section{Correcting palindromes improves read mapping}

Using the BLASR default settings and an additional filter of at least $80 \%$ nucleotide identity between read and reference, both the raw and clean read sets map well (Table 2). Palindromic reads map partially, leaving a (potentially large) proportion of the reads unmapped. This becomes clear when only mappings are considered where $80 \%$ and $95 \%$ of the complete read can be aligned: mapping efficiency for the raw read set drops from 99 to $44 \%$ and finally to $34 \%$. For the clean reads, the mapping rates are $99 \%, 81 \%$ and $66 \%$ respectively, higher than for the noWGA read set $(95 \%, 72 \%$ and $57 \%)$. Average coverages show similar effects. These mappings statistics indicate that the clean reads map more accurately and with higher read coverage than the

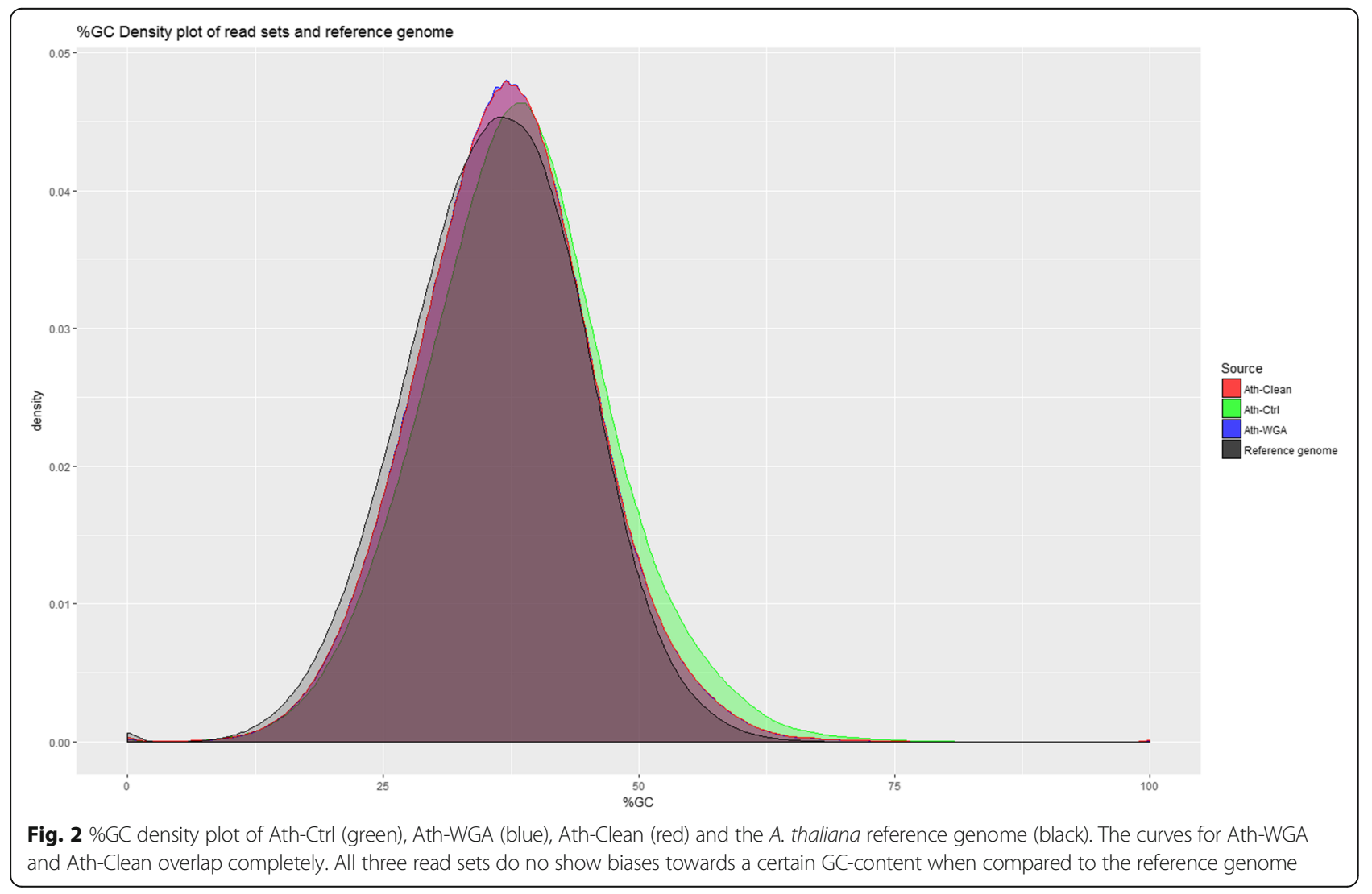


Table 2 Read mapping statistics

\begin{tabular}{|c|c|c|c|c|c|c|c|c|c|}
\hline \multirow[b]{2}{*}{ Alignment filter } & \multicolumn{3}{|c|}{ Reads mapped (\%) } & \multicolumn{3}{|c|}{ Avg. coverage } & \multicolumn{3}{|c|}{ Avg. read length } \\
\hline & - & $80 \%$ & $95 \%$ & - & $80 \%$ & $95 \%$ & - & $80 \%$ & $95 \%$ \\
\hline Ath-WGA & 99 & 44 & 34 & 40.5 & 17.4 & 13.4 & 8987 & 5568 & 5397 \\
\hline Ath-Clean & 99 & 81 & 66 & 55.1 & 48.1 & 41.2 & 4690 & 4532 & 4697 \\
\hline Ath-Ctrl & 95 & 72 & 57 & 34.2 & 29.2 & 24.8 & 5799 & 5583 & 5852 \\
\hline
\end{tabular}

Statistics of read mappings with BLASR to the TAIR10 reference genome, calculated without filtering for a minimum read alignment length ('-') and after filtering for reads aligned with at least $80 \%$ or $95 \%$ nucleotide identity

raw reads do. The complete mapping reports are presented in Additional files 2, 3, 4 .

To verify the palindromic nature of the reads, the locations of the clean reads were also investigated. If the raw reads indeed contain palindromic sequences, the parts of the clean reads should map to the same region in the genome (in contrast to chimeric reads, where the parts originate from different regions in the genome). To verify this, the longest distance between the mapping locations of each part of the corrected reads was calculated and related to the length of the original raw read. 96.5\% of these mapping distances are within the read length of the original read, showing that most of the clean reads map to the same region in the genome and that the original raw reads indeed contain palindromes, not other types of chimeras.

\section{Assembly quality of corrected WGA reads approaches that of non-amplified data}

To assess whether correcting palindromes also benefits assembly, we investigated two realistic scenarios using the A. thaliana data (Ath-WGA, Ath-Clean, Ath-Ctrl): PacBio-only assembly using Canu and a hybrid assembly, combining PacBio and Illumina HiSeq data, using DBG2OLC/Sparse. On the control data set Ath-Ctrl, this results in assemblies with overall good assembly statistics, with DBG2OLC yielding the best results (Table 3). Repeating the process with the original WGA data gives far worse results; the DBG2OLC assembly has, for example, an N50 value about 26-fold smaller than the N50 value of the control data and the assembly covers only about half (49.7\%) of the reference genome.

Correcting the palindromic reads improves the hybrid assembly: although the N50 is lower than that of the Ath-Ctrl assembly, the assembly length and genome coverage are higher.

The Ath-Clean PacBio-only assembly is even better than the assembly based on the Ath-Ctrl data, with a higher N50 and genome coverage (Table 3). This is also reflected by the contig length distribution (Additional file 5). Apparently, the removal of conflicting information outweighs the loss of long-range information.

The hybrid assembly and the PacBio-only assembly based on Ath-Clean are longer than the TAIR10 reference genome (119.7 Mb), being $123.9 \mathrm{Mb}$ and $131.0 \mathrm{Mb}$ respectively. The full genome is thought to be approximately $135 \mathrm{Mb}$ [21], so this additional sequence information could be new genomic data. No further testing has been done to verify this.

De novo assembly based solely on long reads of flow-sorted chromosomes is now possible

After correcting the palindromes in the original gorilla PacBio reads (see section "Discussion".1) we were able to create two PacBio-only assemblies: GorY-WGA based on the raw data set and GorY-Clean, based on the clean

Table 3 Statistics on the de novo assemblies

\begin{tabular}{|c|c|c|c|c|c|c|}
\hline \multirow[b]{2}{*}{ Read set } & \multicolumn{3}{|c|}{ PacBio-only (Canu) } & \multicolumn{3}{|c|}{ Hybrid (DBG2OLC/Sparse) } \\
\hline & Ath-Ctrl (C1) & Ath-WGA (C2) & Ath-Clean (C3) & Ath-Ctrl (D1) & Ath-WGA (D2) & Ath-Clean (D3) \\
\hline No. contigs & 852 & 2128 & 1015 & 476 & 4818 & 1753 \\
\hline Ass. length (Mbp) & 115.6 & 116.8 & 123.9 & 110.9 & 108.9 & 131.0 \\
\hline Longest contig (Kbp) & 1181 & 655 & 3402 & 5667 & 246 & 2239 \\
\hline GC (\%) & 36.0 & 36.2 & 36.12 & 35.97 & 36.57 & 36.21 \\
\hline N50 (Kbp) & 293 & 73 & 302 & 823 & 32 & 278 \\
\hline L50 & 117 & 479 & 109 & 33 & 951 & 113 \\
\hline Covered (\%) & 86.6 & 91.2 & 97.3 & 85.1 & 49.7 & 96.3 \\
\hline Dupl. ratio & 1.09 & 1.07 & 1.06 & 1.08 & 1.34 & 1.13 \\
\hline
\end{tabular}

Statistics on the PacBio-only and hybrid assemblies of the various datasets. Note that the TAIR10 reference genome is $119.7 \mathrm{Mb}$, with the full genome thought to be approximately $135 \mathrm{Mb}[21]$ 
reads. The GorY-WGA assembly was added to the comparison to stay in line with the Arabidopsis thaliana analyses described in the previous section and also to verify that the increase in quality is not only due to a better performing software application. Figure 3 shows the length distributions of both the contigs and the scaffolds in the previously published GorY hybrid assembly and the contigs in the new GorY-Clean / Gory-WGA PacBio-only assemblies. The GorY scaffolds were created by using long-range information to connect the contigs [4]. The scaffolds contain no additional information, except sequence contiguity. Gaps between contigs in the scaffolds are filled with Ns. The top-10 longest contigs of GorY-Clean are as long as the top-10 longest GorY scaffolds, showing that the new contigs already contain the same contiguity except that the gaps are filled with sequence information. The scaffolded GorY assembly seems larger than the GorY-Clean one (Table 4, Additional file 6). However, this is misleading as it contains $2.4 \mathrm{Mbp}$ of Ns; the actual nucleotide content of the GorY assembly is 1.3 Mbp less than that of GorY-Clean. This is corroborated by further assembly statistics (Table 4). In terms of structure, the GorY-Clean assembly resembles the human Y chromosome assembly more than the original assembly (Additional file 7). The GorY-WGA assembly is also of higher quality compared to the GorY contigs, but not as good as the GorY-Clean assembly. We attribute the quality increase of GorY-WGA compared to the GorY assembly to the use of Canu [22]; the improvement of GorY-Clean over GorY-WGA is most likely due to correcting the palindromic reads with Pacasus.
The accuracy of the newly constructed GorY-Clean contigs becomes more apparent when looking at the read mapping statistics (Table 5). To calculate these, only contigs are used as these contain sequence information: reads will not map to Ns in the scaffolds. The gorilla Illumina HiSeq reads map better to the human reference genome (HumY) than to the original GorY assembly (in terms of genome coverage) and overall mapping accuracy is highest for both newly created assemblies. The GorY-Clean assembly is better covered by the read data than the GorY-WGA assembly is, regardless of whether corrected or non-corrected reads are used for evaluation. These results indicate that, apparently, currently available assemblers (in our case Canu) are better at handling chimeric reads than previous software and that the newly created assemblies (GorY-WGA and GorY-Clean) are more accurate than GorY.

The average coverage when using the raw reads increased from $73.15 \mathrm{x}$ to $83.21 \mathrm{x}$ for the GorY-WGA and GorY-Clean respectively and with the corrected reads from 97.08x to $109.67 x$. These results show that the de novo GorY-Clean assembly fits the read data best and, as seen with the Arabidopsis thaliana data, mapping accuracy increases after correcting the palindromic reads.

\section{Resolving artificial duplications provides a higher coverage of genes on the Gorilla Y chromosome}

The gorilla Y chromosome contains 12 single-copy X-degenerate genes [23]. To evaluate completeness of these genes in the assemblies, their corresponding transcript sequences were mapped to the GorY and GorY-Clean

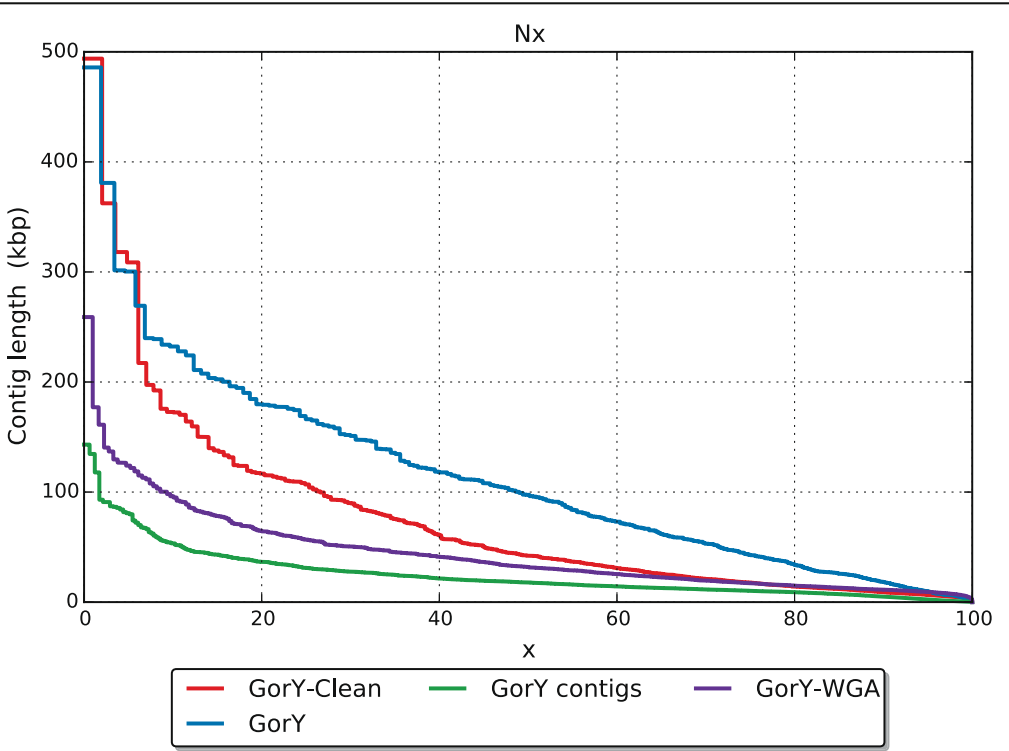

Fig. 3 Contig length distributions. Contig length (y-axis) distribution of the published gorilla Y chromosome (GorY), the contigs underlying this assembly (GorY contigs), the de novo assembly based on raw PacBio data set (GorY-WGA) and of the de novo assembly of the cleaned reads (GorY-Clean). The x-axis shows the fraction of the assembly (e.g. the N20, N50, etcetera) 
Table 4 Human and gorilla Y-chromosome assembly statistics

\begin{tabular}{|c|c|c|c|c|c|c|}
\hline & Assembly size (Mbp) & Ns (Mbp) & Non-Ns (Mbp) & No. sequences & N50 (Kbp) & Longest seq. (Kbp) \\
\hline HumY & 57.2 & 30.4 & 26.8 & 1 & & 57,227 \\
\hline GorY, contigs & 23.0 & 0 & 23.0 & 3001 & 18 & 143 \\
\hline GorY, scaffolds & 25.4 & 2.4 & 23.0 & 697 & 98 & 486 \\
\hline GorY-WGA, contigs & 26.5 & 0 & 26.5 & 1128 & 32 & 256 \\
\hline GorY-Clean, contigs & 24.3 & 0 & 24.3 & 1062 & 42 & 494 \\
\hline
\end{tabular}

Assembly statistics for the published human and gorilla $Y$ chromosome assemblies and the new assemblies

assemblies using the mRNA aligner GMAP [24]. The resulting alignments were subsequently used to identify the contigs/scaffolds that harbor these genes. For these 12 genes, the transcript coverage was on average higher in GorY-Clean contigs (84.9\%) than in GorY scaffolds (74.9\%), while sequence identity was similar (Additional files 8, 9). Additionally, the complete (exons and introns) sequences of the orthologous genes in the human genome (GRCh38) were aligned to the contigs/scaffolds harboring these genes in the GorY and GorY-Clean assemblies. Visual inspection of the dotplots (Additional files 10, 11, 12, 13, 14, 15) identified fewer inversions and duplications in the GorY-Clean contigs than in the GorY scaffolds (Additional file 16). In the alignment of the contigs from the GorY-Clean to the GorY scaffolds containing the same genes (Additional files 17, 18, 19, 20, 21, 22), no inverted duplications were detected in the GorY-Clean sequences. In contrast, numerous inverted duplications were visible in GorY sequences (Additional file 23). Thus, many inverted duplications were resolved in the assembly generated from the sequencing reads corrected by Pacasus, suggesting that such duplications indeed are an artefact of WGA.

\section{Effects on repeats and palindromic sequences of biological origin}

DNA sequences are known to contain many different types and families of repeat sequences [25], including palindrome sequences [26]. These repeat sequences are present in the long reads after sequencing and can be detected by Pacasus as false positive palindromic sequences. When all repeats of biological origin are split, the subsequent de novo assembly will contain only collapsed regions effectively removing the repeats from the assembly. However, we speculate that most if not all of the true repetitive sequence are contained in sufficiently long reads to cover the repeats and therefore will not be considered by Pacasus for correction. In real data sets it is not known a priori which reads contain the true repetitive sequences, but evaluation is possible after performing the de novo assemblies: for both the A. thaliana and the gorilla $\mathrm{Y}$ chromosome the repeat content is known.

Table 6 shows the by RepeatMasker [27] detected repeat content of the A. thaliana assembly, including the reference assembly TAIR10. The repeat content of the assembly based on Ath-Clean (C3) is close to that of the reference and is higher than found in the assemblies based on Ath-Ctrl (C1) and Ath-WGA (C2), which is in line with the overall genome assembly statistics (Table 3).

To study the effect of Pacasus on actual biological palindromes present on the $\mathrm{Y}$ chromosome, 1-kb non-overlapping windows in GorY and GorY-Clean assemblies were identified which have high identity (> $80 \%)$ to well-defined human chromosome Y palindromes P1-P8 (arm lengths 8.7-1450 kb) [28] and X-degenerate gene (XDG) regions. Since the two arms of each palindrome have high identity, the windows representing the palindrome arms should have twice the read depth compared to the windows overlapping with the single-copy XDG regions on the Y chromosome. In order to obtain the read depth of these regions, Illumina-based

Table 5 Read mapping statistics on the human and different GorY assemblies

\begin{tabular}{|c|c|c|c|c|c|c|c|c|c|c|}
\hline \multirow{3}{*}{ Assembly } & \multirow{3}{*}{ Length (Mbp) } & \multicolumn{3}{|l|}{ HiSeq } & \multicolumn{3}{|c|}{ GorY-WGA } & \multicolumn{3}{|c|}{ GorY-Clean } \\
\hline & & \multicolumn{2}{|c|}{ Genome coverage } & \multirow[t]{2}{*}{ Read cov. } & \multicolumn{2}{|c|}{ Genome coverage } & \multirow[t]{2}{*}{ Read cov. } & \multicolumn{2}{|c|}{ Genome coverage } & \multirow[t]{2}{*}{ Read cov } \\
\hline & & (Mbp) & (\%) & & (Mbp) & (\%) & & (Mbp) & (\%) & \\
\hline HumY & 26.8 & 22.3 & 83 & 1897 & 18.2 & 68 & 58.87 & 18.5 & 69 & 74.84 \\
\hline GorY & 23.0 & 18.3 & 80 & 1169 & 21.1 & 92 & 71.33 & 21.1 & 92 & 99.15 \\
\hline GorY-WGA & 26.5 & 24.9 & 94 & 1353 & 26.5 & 100 & 73.15 & 26.5 & 100 & 97.08 \\
\hline GorY-Clean & 24.3 & 22.4 & 92 & 1586 & 24.3 & 100 & 83.21 & 24.3 & 100 & 109.67 \\
\hline
\end{tabular}

Mapping of HiSeq, PacBio WGA and PacBio cleaned reads on the human Y chromosome (HumY), the gorilla Y chromosome (GorY) and the newly created gorilla $Y$ assemblies (GorY-WGA, GorY-Clean). The read coverage is the average number of reads that a nucleotide has aligned to it 
Table 6 Repeat content

\begin{tabular}{ll}
\hline Assembly & Overall repeat content (\% of assembly) \\
\hline Canu Ath-Ctrl (C1) & 15.73 \\
Canu Ath-WGA (C2) & 15.49 \\
Canu Ath-Clean (C3) & 16.76 \\
TAIR10 & 16.88 \\
\hline
\end{tabular}

Repeat content found by RepeatMasker in the different $A$. thaliana assemblies

flow-sorted gorilla $\mathrm{Y}$ paired end reads were aligned to GorY and GorY-clean assembly, and the read depth for the windows specific to palindrome regions and XDG regions were extracted. Figure $4 \mathrm{a}$ and $\mathrm{b}$ show that each palindrome is represented separately and Pacasus decreases the read depth for several palindromes, e.g., P3, P4, and P7. Nevertheless, the median read depth for each palindrome (except for P8) is still higher than XDG for both GorY and GorY-clean. This indicates that the biological palindromes are preserved by Pacasus.

\section{Discussion}

After processing of the long reads, Pacasus has limited effect on the number of nucleotides in the read set and decreases the average read length by less than $50 \%$. A downside of this process is that inverted repeats present in the genome will be treated as chimera, so that the repeat will be split into its separate elements, if the read does not span the full repeat. However, as shown in this manuscript, not all long reads suffer from chimeras. In most cases there will be sufficient reads long enough to cover the inverted repeat and palindrome sequences that are not split by Pacasus as shown in the assessment of the A. thaliana assemblies. It should be noted that $\mathrm{Y}$ chromosomes naturally possess non-artificial palindromes [28] and our analyses show that these palindromic sequences are also present in the de novo assembly after processing the reads by Pacasus.

Flow-sorted chromosomal DNA is usually contaminated with DNA from other chromosomes. Also with the gorilla sample, the original estimate is that approximately a third of the reads originate from the Y chromosome [4]. This is supported by our results, with $1,742,887$ PacBio reads out of 4,546,488 reads mapping to the GorY-Clean assembly (38\%). Consequently, some of the assembled contigs are not part of the gorilla $\mathrm{Y}$ chromosome but are from other chromosomes. Further analyses need to be performed to verify which contigs indeed originate from the Y chromosome. One suggestion is to look at read coverage: high coverage could point to $\mathrm{Y}$ chromosome sequences (see Additional file 7b). The next step to further improve the quality of the assembly could be to scaffold the contigs using the RNA-Seq data from the previous study [4] with for example SSPACE [29] and polish the final assembly with the HiSeq paired-end data using Pilon [30].

Tissue-specific analysis at the genome level is becoming more important in, for example, studying cancer genomes, but for genome assembly approaches they are

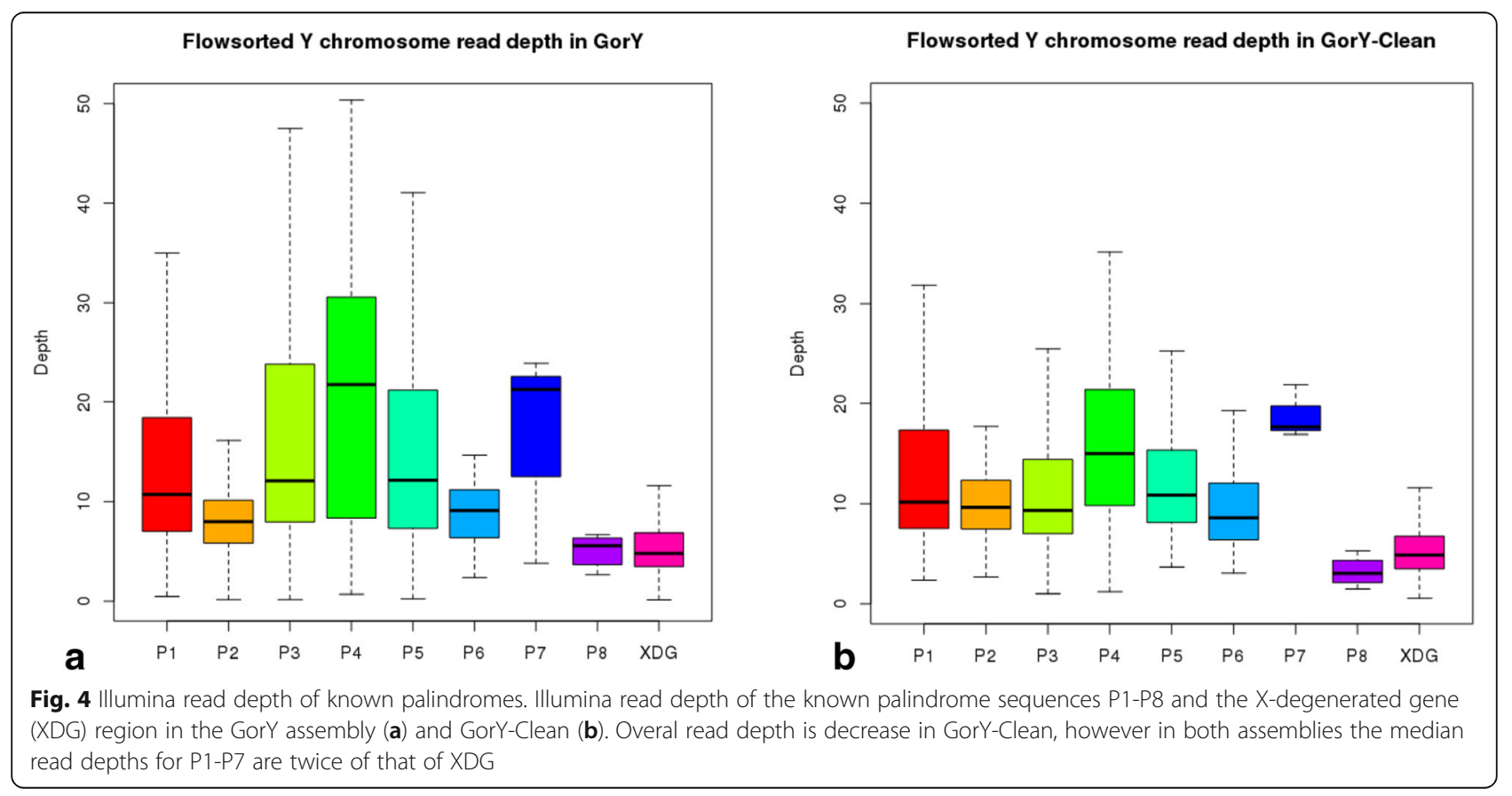


currently limited to short-read sequencing [31] and hence result in more fragmented assemblies than is possible with long reads. For polyploid plant species is possible to select pollen and extract DNA from these cells, effectively decreasing the ploidy by half and therefor also decreasing the complexity of subsequent assembly process. And recent research shows that CRISPR-Cas9 introduces unwanted changes in the genome best detectable by long-read sequencing [32]. By combining WGA and Pacasus on these types of tissues it is now possible to isolate low amounts of DNA and to produce a high-quality genome to find these alterations in the genome.

A possible application not discussed in this paper is the detection of a SMRTbell adapter that is missed by the PacBio software pipeline, producing a raw read with the same structure as created with WGA. These incorrect reads, although perhaps present in low numbers, can have an impact on quality of the de novo assembly. When a non-WGA PacBio dataset with high genome coverage produces a fragmented assembly, it is worthwhile to run Pacasus on this dataset to correct the palindrome sequences created due to the missed SMRTbell adapter.

The detection of the palindrome sequences requires a full Smith-Waterman alignment due to the high error rate of the long-read technologies, which takes a considerable amount of compute power. Using high performance software and several GPUs we were able to process one SMRTcell per day, roughly keeping pace with sequencing speed of the PacBio RSII. The throughput of the PacBio Sequel is higher, hence processing these SMRTcells will require more time or compute resources, but we believe the results presented in this manuscript warrant the investment.

To find the location in the read at which it needs to be split, the backtrace part of the Smith-Waterman alignment algorithm needs to be performed [15]. In the current implementation of the PaSWAS module used for SW, the memory requirements are quadratic in the length of the read. For reads above $100 \mathrm{~kb}$ this memory requirement may limit the use of Pacasus. Currently the PacBio platforms generate reads below this length, but we expect the Oxford Nanopore platforms to go beyond this limit for at least some the reads in the near future. We will continue to work on Pacasus to decrease the memory requirements of the software to ensure that future output of sequencing platforms can be handled properly. The presented settings for the SW alignment are based on the error model of the RSII and our in-house experience with PacBio sequencing read qualities. For application on PacBio Sequel and Oxford Nanopore data, their respective error models may warrant minor changes to these settings.

\section{Conclusions}

Whole-genome amplification is required for sequencing when a biological sample contains insufficient DNA for direct use in library preparation, but the process creates chimeric fragments. We have developed a new method, Pacasus, to correct long, error-rich reads containing such chimeras, based on high-speed Smith-Waterman alignment. We demonstrated the performance of Pacasus in terms of read mapping accuracy and assembly quality, showing that the loss in read length is clearly offset by the removal of incorrect contiguity information. On the Arabidopsis data, the hybrid assembly improves markedly in quality; and on the gorilla data, a PacBio-only assembly on clean reads is even of higher quality than a hybrid assembly including the original reads. The differences between the GorY-Clean and GorY-WGA assemblies are, however, not as large as in the $A$. thaliana case. The underlying reason for this is the much lower number of detectable palindromes in the gorilla read set: $11.8 \%$ of the reads contain palindromes, compared to $45.8 \%$ of the reads in the $A$. thaliana set. Correcting the relatively low number of reads containing palindromes in the gorilla data set already gave an improvement in assembly statistics, which indicates that the impact of these incorrect reads on the assembly quality is high. We expect that longer reads contain more palindromes, as indicated by the differences in average lengths before and after correcting in both examples.

In summary, Pacasus now allows to analyze PacBio data obtained from low amounts of DNA, making it possible to apply the power of long read technology to, for example, the study of single cells (e.g. in cancer research) and the study of single chromosomes (also in polyploid organisms).

\section{Methods}

\section{The Pacasus algorithm}

To detect chimeras created during WGA, raw PacBio reads are aligned to their reverse-complement sequence with Smith-Waterman (Fig. 1) using pyPaSWAS [16, 33]. The parameters used for alignment are: gap score, -3 ; match score, 3 ; mismatch score, -4 . For filtering, the parameters are: filter factor, 0.01 ; query coverage, 0.01 ; query identity, 0.01; relative score, 0.01; and base score, 1.0. When no overlap is found in the alignment, the read is left intact and stored in the output file; otherwise the read is split at the center of alignment (see Fig. 1(d)). Both resulting fragments are again processed as if they were original reads, to allow the detection of nested palindrome sequences, until no overlap is detected anymore. If a fragment becomes shorter than a minimum length (default $50 \mathrm{bp}$ ) it is discarded. Note that the nucleotide information in the reads is neither removed nor changed. Pacasus is implemented in Python 2.7 and, besides pyPaSWAS (version > $>2.0$ ), depends on Biopython [34] 
(version > = 1.67), numpy (http://www.numpy.org/) (version > $=1.8 .0)$ and scipy [35] (version $>=0.12 .0)$.

\section{Data for Arabidopsis evaluation}

DNA was isolated from two Arabidopsis thaliana plants, labeled "Ath-WGA1" and "Ath-WGA2", and amplified using the REPLI-g Mini Kit (QIAGEN Benelux BV, Venlo, The Netherlands). The Arabidopsis thaliana are in-house samples based on low-input plant materials (for more details on DNA isolation, library preparation and sequencing see [36]). Both samples were sequenced on both an Illumina HiSeq2000 sequencer and a PacBio RSII sequencer. A third DNA sample was used to generate PacBio RSII data without WGA ("Ath-Ctrl"). Table 7 shows the number of reads generated by each platform and for each sample. To evaluate mapping performance, PacBio reads were mapped to the TAIR10 Arabidopsis thaliana Columbia reference genome (http://www.arabi dopsis.org) using BLASR version 1.3.1.124201 [37], and alignments with identity $<80 \%$ were filtered out by a Python script. Mapping reports were generated in CLCBio version 8.0.2 (http://www.clcbio.com).

PacBio-only de novo assemblies of the A. thaliana genome were created using Canu version 1.3 [22]. Hybrid assemblies, combining the HiSeq2000 and RSII data, were created with DBG2OLC (released in 2016) [38]. DBG2OLC requires as input a HiSeq-only assembly; which we created using Sparse (released in 2015) [39], as recommended on the website by the authors of DGB2OLC, based on the HiSeq data from the WGA samples in all cases. The assembly was finalized with the PacBio reads.

We combined Ath-WGA1 and Ath-WGA2 into a single set, Ath-WGA, and created assemblies combining the HiSeq contigs with the raw Ath-WGA reads, with corrected (or 'clean') Ath-WGA reads (Ath-Clean) and with Ath-Ctrl reads. To evaluate quality, assemblies were compared to the reference genome using QUAST version $4.3[40]$.

\section{Data for the gorilla $Y$ chromosome evaluation}

PacBio RSII data of a flow-sorted and amplified gorilla $Y$ chromosome, GorY-WGA (Table 1), was downloaded from the NCBI Short Read Archive (SRA SRX1161235).
The previously published assembly of the gorilla Y chromosome and the publicly available data from the flow-sorted, whole-genome amplified and de-branched gorilla Y chromosome, GorY [4], were downloaded as well (GCA_001484535.2). Canu version 1.3 was used for the assembly of the PacBio reads. For comparison, the human chromosome Y assembly (NC_000024.10), HumY, was downloaded. QUAST [40] was used for assembly comparison and statistics. PacBio reads were mapped to HumY, GorY and the new assemblies using BLASR ( $>80 \%$ identity and $>80 \%$ read coverage); Illumina HiSeq 2500 PE reads (SRA SRR2176191) were mapped using CLCBio version 8.0.2. Statistics for all mapping results were calculated in CLCBio. For calculating the contig length distribution of the GorY assembly, scaffolds were broken up and N's were removed. The gorilla X-degenerate gene transcripts were retrieved from a previous study [23]. GMAP version 2017-03-17 [24] was used to align the transcripts to the assemblies.

\section{Repeats and palindrome detection}

RepeatMasker [27] was configured with rmblastn (2.2.27+) [41, 42] and RepBase (20140131) [43] for masking the $A$. thaliana assemblies and TAIR10 reference genome.

Following Tomaszkiewicz et al. [4], the gorilla Y contigs were broken into $1-\mathrm{kb}$ windows and each window was aligned to human reference hg38 using lastz [44] (--scores $=$ human_primate.q, $\quad$--seed $=$ match12, $\quad$--markend). RepeaMasker was also run on gorilla Y contigs to mask repeats and later for each window the total number of masked sites ' $\mathrm{N}$ ' within a window were calculated. The windows which overlap with human Y chromosome palindromes and XDG genes were identified and filtered to make sure that they have at least $80 \%$ match with the human reference and less than $20 \%$ of N's throughout the window.

BWA mem [45] was used to align the flow-sorted gorilla Y paired-end reads (SRX1160374) to the GorY-clean and GorY assemblies (unmasked). The bedtools [46] coverage function was used to calculate the read depth and coverage of each window. If the windows had $>80 \%$ coverage they were used to create boxplots within their respective palindromes. The boxplots were generated using $\mathrm{R}$ boxplot command with outline $=$ TRUE parameter set.

Table 7 Datasets used for the performance analysis of Pacasus

\begin{tabular}{|c|c|c|c|c|c|c|}
\hline \multirow[t]{2}{*}{ Species } & \multirow[t]{2}{*}{ Sample } & \multirow[t]{2}{*}{ WGA } & \multicolumn{2}{|c|}{ Illumina HiSeq2000 } & \multicolumn{2}{|c|}{ PacBio RSII } \\
\hline & & & reads & length & Reads & avg. Length \\
\hline \multirow[t]{3}{*}{ Arabidopsis thaliana } & Ath-WGA1 & yes & $31,233,196$ & 100 & 462,138 & 9326 \\
\hline & Ath-WGA2 & yes & $43,810,780$ & 100 & 447,364 & 8544 \\
\hline & Ath-Ctrl & no & & & 940,162 & 5680 \\
\hline Gorilla gorilla & GorY-WGA & yes & $279,601,852$ & 150 & $3,596,236$ & 5468 \\
\hline
\end{tabular}


The Human_primate.q file used for primate to primate alignments in lastz is as below:

$$
\begin{aligned}
& \text { gap_open_penalty }=500 \# \mathrm{O} \\
& \text { gap_extend_penalty }=30 \# \mathrm{E} \\
& \text { hsp_threshold }=3000 \# \mathrm{~K} \\
& \text { gapped_threshold }=4500 \# \mathrm{~L} \\
& \text { x_drop }=900 \# \mathrm{X} \\
& \text { y_drop } \quad=15000 \# \mathrm{Y}
\end{aligned}
$$

\section{A C G T}

\section{A $90-330-236-356$ \\ C-330 100-318-236 \\ G-236-318 100-330 \\ T-356-236-330 90}

\section{Additional files}

Additional file 1: Number of Pacasus iterations per read in the cleaned data set. (DOCX $40 \mathrm{~kb}$ )

Additional file 2: Mapping Report for Ath-Ctrl BLASR-mapping. (PDF 427 kb) Additional file 3: Mapping Report for Ath-Clean BLASR-mapping. (PDF $418 \mathrm{~kb}$ )

Additional file 4: Mapping Report for Ath-WGA BLASR-mapping (PDF $416 \mathrm{~kb}$ )

Additional file 5: Cumulative lengths of the PacBio-only and hybrid assemblies of Ath-Ctrl, Ath-WGA and Ath-Clean. (DOCX $114 \mathrm{~kb}$ )

Additional file 6: Cumulative length distribution of gorilla $Y$ chromosome assemblies. (DOCX $264 \mathrm{~kb}$ )

Additional file 7: Mummerplots of HumY against the different GorY assemblies. (DOCX $166 \mathrm{~kb}$ )

Additional file 8: Coverage and identity of the $X$-degenerate gene transcripts in GorY and GorY-Clean assemblies. (DOCX $25 \mathrm{~kb}$ )

Additional file 9: Comparison of published GorY assembly to GorY-Clean in X-degenerate genes. (DOCX $34 \mathrm{~kb}$ )

Additional file 10: Dotplots mapping Human SRY gene to the GorY scaffold and GorY-Clean contig. (DOCX $39 \mathrm{~kb}$ )

Additional file 11: Dotplots mapping Human AMELY gene to the GorY scaffold and GorY-Clean contig. (DOCX $40 \mathrm{~kb}$ )

Additional file 12: Dotplots mapping Human NLGN4Y gene to the GorY scaffolds and GorY-Clean contigs. (DOCX 204 kb)

Additional file 13: Dotplots mapping Human PRKY gene to the GorY scaffolds and GorY-Clean contigs. (DOCX 228 kb)

Additional file 14: Dotplots mapping Human USP9Y gene to the GorY scaffolds and GorY-Clean contigs. (DOCX 186 kb)

Additional file 15: Dotplots mapping Human ZFY gene to the GorY scaffold and GorY-Clean contig. (DOCX 46 kb)

Additional file 16: The number of duplication or inversion events that were observed on contigs/scaffolds when aligned to human X-degenerate genes. (DOCX 26 kb)

Additional file 17: Dotplots mapping KDM5D gene containing GorY scaffold to GorY-Clean contig. (DOCX $39 \mathrm{~kb}$ )

Additional file 18: Dotplots mapping TBL1Y gene containing GorY scaffolds to GorY-Clean contigs. (DOCX $162 \mathrm{~kb}$ )

Additional file 19: Dotplots mapping USP9Y gene containing GorY scaffolds to GorY-Clean contigs. (DOCX $134 \mathrm{~kb}$ )

Additional file 20: Dotplots mapping SRY gene containing GorY scaffold to GorY-Clean contig. (DOCX $47 \mathrm{~kb}$ )
Additional file 21: Dotplots mapping TMSB4Y gene containing GorY scaffold to GorY-Clean contig. (DOCX $59 \mathrm{~kb}$ )

Additional file 22: Dotplots mapping DBY gene containing GorY scaffold to GorY-Clean contig. (DOCX $70 \mathrm{~kb}$ )

Additional file 23: The number of inverted duplication events visually observed on XDG-containing contigs/scaffolds when they are aligned to one another in a dotplot. (DOCX $26 \mathrm{~kb}$ )

\section{Abbreviations}

Ath: Arabidopsis thaliana; GorY: Gorilla gorilla Y chromosome; HMW: High Molecular Weight; MP: Mate-pair; PE: Paired-End; WGA: Whole Genome Amplification; XDG: X-Degenerated Gene

\section{Acknowledgments}

We would like to thank Jan-Peter Nap (Hanze University of Applied Sciences, Groningen) for his comments and suggestions on the text.

\section{Funding}

This work was carried out on the Dutch national e-infrastructure with the support of SURF Cooperative. The authors acknowledge support from the National Science Foundation (awards DBI-ABI 0965596 to K.D.M.; DBI-1356529, IIS-1453527, IIS-1421908, and CCF-1439057 to P.M.), Eberly College of Sciences and Institute for CyberScience at Penn State, the Penn State Clinical and Translational Sciences Institute, and the Pennsylvania Department of Health, using Tobacco Settlement Funds (the Department specifically disclaims responsibility for any analyses, interpretations or conclusions). RV was supported by the National Institutes of Health (NIH)-PSU funded Computation, Bioinformatics and Statistics (CBIOS) Predoctoral Training Program (1T32GM102057-0A1).

\section{Availability of data and materials}

Project name: Pacasus.

Project home page: https://github.com/swarris/Pacasus

Archived version: DOl: https://doi.org/10.5281/zenodo.1148798

Operating system(s): Platform independent.

Programming language: Python

Other requirements: CUDA and/or OpenCL.

License: MIT.

The datasets supporting the conclusions of this article are available at the European Nucleotide Archive under accession number PRJEB21791. The Arabidopsis thaliana HiSeq read sets are available under accessions ERX2095148 and ERX2095149. The PacBio data sets are available under accessions ERX2095150 and ERX2095151. The Gorilla Y chromosome assembly has been assigned accession number GCA_900199665.

\section{Authors' contributions}

SW implemented the Pacasus tool and wrote the draft manuscript together with and supervised by DdR. ES was responsible for setting up the ATH experiments, including the whole-genome amplification of the DNA and identifying the palindrome formation after WGA. TH and BtLH sequenced the ATH samples using the PacBio and Illumina HiSeq platform respectively. SW and HvdG performed the de novo assemblies on the ATH data set. SW, ES, HG and GSP were involved in validating the results after correction of the palindrome sequences and in checking the quality of the ATH assemblies. SW created the PacBio-only assemblies of the gorilla Y chromosome. RV, PM and KM did the comparison to the existing gorilla Y chromosome assembly. All authors read and approved the manuscript.

Ethics approval and consent to participate Not applicable.

\section{Consent for publication}

Not applicable.

\section{Competing interests}

GSP and HvdG moved to Genetwister Technologies BV after contributing to this research. Genetwister technologies BV is not affiliated with this research and is not financially or otherwise linked to the project. The authors declare that they have no competing interests. 


\section{Publisher's Note}

Springer Nature remains neutral with regard to jurisdictional claims in published maps and institutional affiliations.

\begin{abstract}
Author details
${ }^{1}$ Applied Bioinformatics, Wageningen University and Research, Wageningen, The Netherlands. ${ }^{2}$ Bioinformatics Group, Wageningen University and Research, Wageningen, The Netherlands. ${ }^{3}$ Bioinformatics and Genomics Graduate Program, Pennsylvania State University, University Park, State College, PA 16802, USA. ${ }^{4}$ Computation, Bioinformatics, Statistics Graduate Training Program, Pennsylvania State University, University Park, State College, PA 16802, USA. ${ }^{5}$ The Center for Computational Biology and Bioinformatics, Pennsylvania State University, University Park, State College, PA 16802, USA. 'Department of Computer Science and Engineering, Pennsylvania State University, University Park, State College, PA 16802, USA. ${ }^{7}$ Department of Biochemistry and Molecular Biology, Pennsylvania State University, University Park, State College, PA 16802, USA. ${ }^{8}$ The Center for Medical Genomics, Pennsylvania State University, University Park, State College, PA 16802, USA. ${ }^{9}$ Department of Biology, Pennsylvania State University, University Park, State College, PA 16802, USA. ${ }^{10}$ Present address Genetwister Technologies BV, Wageningen, The Netherlands.
\end{abstract}

Received: 7 August 2018 Accepted: 15 October 2018 Published online: 06 November 2018

\section{References}

1. HPJ B, den Dunnen JT. Next generation sequencing technology: advances and applications. Biochim Biophys Acta - Mol Basis Dis. 2014;1842:1932-41.

2. Shapiro E, Biezuner T, Linnarsson S. Single-cell sequencing-based technologies will revolutionize whole-organism science. Nat. Rev. genet. Nature publishing group, a division of Macmillan publishers limited. All Rights Reserved. 2013;14:618-30.

3. Gawad C, Koh W, Quake SR. Single-cell genome sequencing: current state of the science. Nat Rev Genet Nature Research. 2016;17:175-88.

4. Tomaszkiewicz M, Rangavittal S, Cechova M, Sanchez RC, Fescemyer HW, Harris R, et al. A time- and cost-effective strategy to sequence mammalian $Y$ chromosomes: an application to the de novo assembly of gorilla $Y$. Genome Res Cold Spring Harbor Laboratory Press. 2016;26:530-40.

5. Czyz ZT, Kirsch S, Polzer B. Principles of whole-genome amplification. Methods Mol Biol. 2015;1347:1-14.

6. Lasken RS, Stockwell TB. Mechanism of chimera formation during the multiple displacement amplification reaction. BMC Biotechnol BioMed Central. 2007;7:19

7. Sabina J, Leamon JH. Bias in whole genome amplification: causes and considerations. Methods Mol Biol. 2015;1347:15-41.

8. Zhang K, Martiny AC, Reppas NB, Barry KW, Malek J, Chisholm SW, et al. Sequencing genomes from single cells by polymerase cloning. Nat. Biotechnol. Nat Publ Group. 2006;24:680.

9. Edgar RC, Haas BJ, Clemente JC, Quince C, Knight R. UCHIME improves sensitivity and speed of chimera detection. Bioinformatics. 2011;27:2194-200.

10. Rhoads A, Au KF. PacBio sequencing and its applications. Genomics Proteomics Bioinformatics Elsevier. 2015;13:278-89.

11. Jain M, Olsen HE, Paten B, Akeson M. The Oxford Nanopore MinION: delivery of nanopore sequencing to the genomics community. Genome Biol. 2016;17:239.

12. Hackl T, Hedrich R, Schultz J, Förster F. Proovread: large-scale high-accuracy PacBio correction through iterative short read consensus. Bioinformatics. Oxford University Press. 2014;30:3004-11.

13. Koren S, Schatz MC, Walenz BP, Martin J, Howard JT, Ganapathy G, et al. Hybrid error correction and de novo assembly of single-molecule sequencing reads. Nat Biotechnol Nature Research. 2012;30:693-700.

14. Lee H, Gurtowski J, Yoo S, Marcus S, McCombie WR, Schatz M. Error correction and assembly complexity of single molecule sequencing reads. bioRxiv. Cold Spring Harbor Labs Journals. 2014:006395.

15. Warris S, Yalcin F, Jackson KJL, Nap JP. Flexible, fast and accurate sequence alignment profiling on GPGPU with PaSWAS. Zhang M, editor. PLoS One. 2015;10:e0122524.

16. Warris $S$, Timal NRN, Kempenaar M, Poortinga AM, van de Geest $H$, Varbanescu AL, et al. pyPaSWAS: Python-based multi-core CPU and GPU sequence alignment. PLoS One. 2018:13.
17. Human Genome Sequencing Consortium International. Finishing the euchromatic sequence of the human genome. Nature. Nature Publishing Group; 2004;431:931-45.

18. Rangavittal S, Harris RS, Cechova M, Tomaszkiewicz M, Chikhi R, Makova KD, et al. RecoverY: K-mer based read classification for Y-chromosome specific sequencing and assembly. Bioinformatics. 2017;

19. Chin C-S, Alexander DH, Marks P, Klammer AA, Drake J, Heiner C, et al. Nonhybrid, finished microbial genome assemblies from long-read SMRT sequencing data. Nat. Methods. Nature publishing group, a division of Macmillan publishers limited. All Rights Reserved. 2013;10:563-9.

20. Berlin K, Koren S, Chin C-S, Drake JP, Landolin JM, Phillippy AM, et al. Assembling large genomes with single-molecule sequencing and localitysensitive hashing. Nat Biotechnol BioMed Central. 2015;33:623-30.

21. Schmuths H, Meister A, Horres R, Bachmann K. Genome size variation among accessions of Arabidopsis thaliana. Ann Bot Oxford University Press. 2004;93:317-21.

22. Koren S, Walenz BP, Berlin K, Miller JR, Bergman NH, Phillippy AM. Canu: scalable and accurate long-read assembly via adaptive $k$-mer weighting and repeat separation. Genome Res. 2017;27:722-36.

23. Cortez D, Marin R, Toledo-Flores D, Froidevaux L, Liechti A, Waters PD, et al. Origins and functional evolution of $Y$ chromosomes across mammals. Nature. Nature publishing group, a division of Macmillan publishers limited. All Rights Reserved. 2014;508:488-93.

24. Wu TD, Watanabe CK. GMAP: a genomic mapping and alignment program for mRNA and EST sequences. Bioinformatics. 2005;21:1859-75.

25. Qian Z, Adhya S. DNA repeat sequences: diversity and versatility of functions. Curr Genet. 2017;63:411-6.

26. Inagaki H, Kato T, Tsutsumi M, Ouchi Y, Ohye T, Kurahashi H. Palindromemediated translocations in humans: a new mechanistic model for gross chromosomal rearrangements. Front. Genet. Frontiers Media SA. 2016;7:125.

27. Smit AFA, Hubley R, Green P. RepeatMasker. Available from: http:// repeatmasker.org

28. Skaletsky H, Kuroda-Kawaguchi T, Minx PJ, Cordum HS, Hillier L, Brown LG, et al. The male-specific region of the human $Y$ chromosome is a mosaic of discrete sequence classes. Nature. 2003;423:825-37.

29. Boetzer M, Henkel CV, Jansen HJ, Butler D, Pirovano W. Scaffolding preassembled contigs using SSPACE. Bioinformatics. 2011;27:578-9.

30. Walker BJ, Abeel T, Shea T, Priest M, Abouelliel A, Sakthikumar S, et al. Pilon: an integrated tool for comprehensive microbial variant detection and genome assembly improvement. Wang J, editor. PLoS One. Public Library of Science; 2014;9.

31. Nakagawa H, Fujita M. Whole genome sequencing analysis for cancer genomics and precision medicine. Cancer Sci Wiley/Blackwell (101111). 2018;109:513-22.

32. Kosicki M, Tomberg K, Bradley A. Repair of double-strand breaks induced by CRISPR-Cas9 leads to large deletions and complex rearrangements. Nat. Biotechnol: Nature Publishing Group; 2018.

33. Warris S, Timal R. pyPaSWAS 2016. Available from: https://doi.org/10.5281/ zenodo. 51155

34. Cock PJA, Antao T, Chang JT, Chapman BA, Cox CJ, Dalke A, et al. Biopython: freely available Python tools for computational molecular biology and bioinformatics. Bioinformatics. 2009;25:1422-3.

35. Jones E, Oliphant T, Peterson P. SciPy: open source scientific tools for Python [internet]. 2001. Available from: http://www.scipy.org

36. Schouten HJ, vande Geest H, Papadimitriou S, Bemer M, Schaart JG, MJM S, et al. Re-sequencing transgenic plants revealed rearrangements at T-DNA inserts, and integration of a short T-DNA fragment, but no increase of small mutations elsewhere. Plant cell rep. Springer Berlin Heidelberg. 2017:36:493-504.

37. Chaisson MJ, Tesler G. Mapping single molecule sequencing reads using basic local alignment with successive refinement (BLASR): application and theory. BMC Bioinformatics BioMed Central. 2012;13:238.

38. Ye C, Hill C, Ruan J, Zhanshan, Ma. DBG2OLC: efficient assembly of large genomes using the compressed overlap graph. 2014;

39. Ye C, Ma ZS, Cannon CH, Pop M, Yu DW. Exploiting sparseness in de novo genome assembly. BMC bioinformatics. BioMed Central. 2012;13 Suppl 6:S1.

40. Gurevich A, Saveliev V, Vyahhi N, Tesler G. QUAST: quality assessment tool for genome assemblies. Bioinformatics Oxford University Press. 2013;29:1072-5.

41. Smit A, Hubley R. rmblast . Available from: http://www.repeatmasker.org/ RMBlast.html 
42. Camacho C, Coulouris G, Avagyan V, Ma N, Papadopoulos J, Bealer K, et al. BLAST+: architecture and applications. BMC bioinformatics. BioMed Central. 2009;10:421.

43. Bao W, Kojima KK, Kohany O. Repbase update, a database of repetitive elements in eukaryotic genomes. Mob. DNA. BioMed Central. 2015;6:11.

44. Harris RS. Improved pairwise alignment of genomic DNA. 2007;

45. Li H. Aligning sequence reads, clone sequences and assembly contigs with BWA-MEM. 2013;

46. Quinlan AR, Hall IM. BEDTools: a flexible suite of utilities for comparing genomic features. Bioinformatics. Oxford University Press. 2010;26:841-2.

Ready to submit your research? Choose BMC and benefit from:

- fast, convenient online submission

- thorough peer review by experienced researchers in your field

- rapid publication on acceptance

- support for research data, including large and complex data types

- gold Open Access which fosters wider collaboration and increased citations

- maximum visibility for your research: over $100 \mathrm{M}$ website views per year

At $\mathrm{BMC}$, research is always in progress.

Learn more biomedcentral.com/submissions 